\title{
Burnout and grit in student physical therapists before and after terminal clinical education experiences: a prospective longitudinal study of a single class-year cohort
}

Jennifer C. Kish"*, Jessica L. Dugan² and Peggy B. Gleeson³

'Bowling Green State University, Bowling Green, OH; 'Evidence in Motion, LLC, San Antonio, TX; ${ }^{3}$ Texas Woman's University, Houston, TX

Abstract

Rationale: Burnout has been reported by graduate healthcare students during several phases of preprofessional education. The purpose of this study was to explore changes in levels of Doctor of Physical Therapy (DPT) student burnout and grit before and after clinical education. Additional aims included exploring how burnout and grit might differ based on student gender and over time and what relationships might exist between burnout and grit in this population.

Methods: All enrolled third year DPT students $(n=50)$ at a single physical therapist education program participated in this study. Participants were surveyed using the Maslach Burnout Inventory Human Services Survey and the 12-Item Grit Scale at the end of curricular Year 3 and at graduation after terminal clinical education experiences. Descriptive, exploratory, and comparative statistical methods were used.

Results: Male participants reported significantly decreased emotional exhaustion (EE) from Year 3 to graduation, $p \leq 0.0001$. Overall, the cohort reported significantly decreased EE, $\mathrm{F}(1,48)=12.35, p=0.001, d=0.44$ and increased personal accomplishment, $\mathrm{F}(1,48)=13.322, p=0.001, d=0.58$ after terminal clinical experiences. The main effect of time on grit scores was also significant, $p=0.035$. A moderate inverse relationship existed between student grit levels at Year 3 and EE levels at graduation, $r=-0.447, p=0.01$.

Conclusions: Cohort burnout scores did not meet the Maslach Burnout Inventory criteria definition for burnout; mean EE and personal accomplishment subscale scores significantly changed over time. Male DPT students reported moderate levels of EE at the start of terminal clinical experiences that dropped significantly to low levels compared to female students. Student grit levels in this study were high compared to recent investigations of other DPT cohorts. Grit may have a protective effect against DPT students experiencing burnout.

Meshterms: Burnout, Professional; Physical Therapists/education; Achievement.

Keywords: physical therapist education; clinical education; burnout; grit

Received: 2 February 2021; Revised: 7 September 2021; Accepted: 9 September 2021; Published: 09 November 2021

$\mathrm{B}$ urnout in healthcare providers has been associated with adverse patient outcomes, effects on the healthcare workforce, and increased cost to organizations and systems. ${ }^{1-3}$ Burnout has been described as a work-related syndrome with emotionally intense work demands exceeding one's capacity or resources to manage them. ${ }^{1}$ Burnout has been negatively associated with safety-related quality of care. ${ }^{2,3}$ Burnout in healthcare providers and providers-in-training has been reported to exceed a prevalence of $50 \%{ }^{1}$ Burnout in graduate healthcare students has been well-documented. ${ }^{4,5}$

Burnout has the potential to impact student mental health and professional behaviors development., ${ }^{4,6}$ Burnout is commonly assessed using self-report instruments that evaluate constructs or factors, including perceived levels and frequency of emotional exhaustion (EE), 
depersonalization, personal accomplishment, and engagement. ${ }^{7,8}$ Several factors including increased stress, decreased support from the program/family/friends, progression to later years of training, and female gender (inconclusively) have also been found to be associated with graduate healthcare student burnout. ${ }^{4}$ Student physical therapists participating in clinical education have also described expectations for a dual identity as both a clinician and student. ${ }^{9}$

The impact of setting and work factors on the presence and degree of burnout has been studied in physical therapists and student physical therapists at both the undergraduate and graduate levels. ${ }^{5,10,11}$ In a 2018 cross-sectional study of one, two-campus physical therapist education program (PTEP), Williams et al. found that female Doctor of Physical Therapy (DPT) students reported higher levels of depression and anxiety compared with male students, and older students ( $>29$ years old) reported higher levels of emotional distress and depression when compared with those DPT students $<29$ years old. ${ }^{5}$ Additionally, authors noted that students in the second year of the DPT program reported higher scores across a semester on depression, stress, and disengagement measures than students in didactic year one. ${ }^{5}$

Perseverance, grit, and resilience may be positive adaptations to adversity in healthcare providers and students. ${ }^{12}$ Grit has been investigated in several academic settings, and the study of grit in DPT and other health professional students is emerging. ${ }^{13-16}$ Grit is commonly measured using self-report instruments that assess perceived conscientiousness and an individual's ability to maintain effort and interest over long periods of time despite setbacks. ${ }^{17}$ Grit research in healthcare providers and students has focused on the ability to foster grit or as a tool to assess non-cognitive applicant traits and predict attrition or success in education. ${ }^{14,15}$ Bliss and Jacobson reported a moderate correlation between grit in entry-level DPT students and cumulative undergraduate GPA scores. ${ }^{16}$ Richardson et al. also found a similar moderate correlation between grit and graduate DPT program cumulative GPA. ${ }^{18}$ In 2021, Huhn et al. reported no significant associations between grit and first-year DPT student GPA. ${ }^{19}$ The use of grit scores as a non-cognitive metric in the PTEP admissions process was investigated by Carp et al. who described applicant scores in a cohort study as high and moderately positively correlated $(r=0.553, p<0.01)$ to final GPA in the program..$^{20}$

DPT students have reported increasing levels of exhaustion and disengagement from coursework demands over an academic semester. ${ }^{5}$

Changes in burnout and grit in student physical therapists in the clinical education (vs. academic) setting and the relationships between the two constructs have yet to be fully explored. Although burnout has been studied over the course of a didactic academic semester in a PTEP, little is known about how longer time during clinical education might influence student responses. ${ }^{5}$ The concurrent coursework demands of a PTEP coupled with the role as a clinician increases demands, which may contribute to even greater levels of burnout during the final terminal clinical education experiences. In a separate study of medical students by Jumat et al., grit was reported to have a protective effect against experiencing burnout (odds ratio, 0.84; 95\% CI, 0.74 to 0.96$).{ }^{22}$ Relationships between grit and burnout were explored by Cortez et al. in a longitudinal study of surgical physician residents, and surgeons with burnout had significantly lower grit scores than surgeons without burnout. ${ }^{21} \mathrm{EE}$ was found to peak in the first and third clinical years of residency while feelings of personal accomplishment peaked in the third clinical year. ${ }^{21}$

The purpose of this study was to explore changes in levels of DPT student grit and burnout before and after their last consecutive (terminal) clinical education experiences. Given reported differences in anxiety, stress, and exhaustion between male and female DPT students, we speculated that significant gender differences may also exist in the exhaustion and depersonalization burnout domains at both time intervals. ${ }^{4-6}$ Studies describing grit in DPT students and how it might differ between men and women are limited. ${ }^{13-19}$ Therefore, additional objectives of this study included exploring how burnout and grit might differ based on student gender and over time. We also expected to find significant correlations between burnout and grit variables based on recent investigations. ${ }^{20,21}$

\section{Methods}

This was a prospective, longitudinal, repeated measures cohort study using survey measures with a single class year convenience sample of DPT students from the University of the Incarnate Word, a private PTEP located in the Southwest region of the United States. Students in the population of interest were enrolled in a 3-year, 8-semester onsite DPT program that used a problem-based learning (PBL) curricular model with 44 weeks of clinical education built into four clinical experiences. The first clinical education experience was a 6-week experience at the end of curricular year one (Semester 3); the second was an 8-week clinical experience at the end of Semester 5; students then finished the program with back-to-back third (16 weeks) and fourth (14 weeks) clinical experiences before graduation. Students were responsible for concurrent monthly online content and assignments throughout their terminal clinical education experiences and clinical education course.

\section{Participants}

All third year DPT students $(n=50)$ enrolled at the institution in 2016 were eligible for recruitment into this study. Students were recruited and invited to participate at the end of the sixth and final didactic semester of the program, curricular year 3 (Y3), right before clinical 
experiences 3-4 (terminal experiences). Participants were included in the study if they had signed an informed consent at matriculation into the program as part of the School of Physical Therapy's blanket programmatic Institutional Review Board (IRB) approval titled, Learning Outcomes of a Problem Based Learning Doctor of Physical Therapy Program. Burnout and grit survey instruments were added to the programmatic teaching and learning IRB (\#12-08-003) as an addendum and approved for use. At recruitment, participants were reminded that they could withdraw their programmatic informed consent at any time. No remuneration or incentives were provided as inducement for participation, and students were advised that their participation would not impact academic standing or relationships with faculty in any way.

\section{Instruments}

The Maslach Burnout Inventory-Human Services Survey (MBI-HSS) is the original and most widely used and validated version of the MBI, considered the gold standard for measuring dimensions of burnout in human service professionals including nurses, social workers, and mental health workers. ${ }^{7}$ The MBI assesses three domains of burnout including EE, depersonalization, and personal accomplishment and is available in survey versions adapted and validated for medical personnel (MBI-HSS(MP)), educators (MBI-ES), other workers/occupational groups with the general survey (MBI-GS), and students (MBI-GS(S)). ${ }^{7}$ Given our study's aim to assess DPT student burnout before and after terminal clinical education experiences where the students' primary role was that of preprofessional, we felt that the MBI-HSS version best supported our objectives and allowed for future generalizability and comparison.

The MBI is a 22-item Likert-scale survey. Participants rate statements of job-related feelings based on the intensity and frequency they experience them from 0 (Never) to 6 (Every day). The EE subscale assesses respondent feelings of exhaustion and being emotionally overextended by their work. The DP domain describes an unfeeling impersonal response toward the recipients of a respondent's care, and PA measures respondent feelings of competence and success in their work. ${ }^{1,7}$ The MBI-HSS has acceptable internal consistency, Cronbach's coefficient alpha $=0.83$ for frequency and 0.84 for intensity and reliability for the whole instrument, 0.83 (frequency) and 0.84 (intensity) and subscales ranging from 0.57 to 0.89 .7 The EE subscale includes nine questions for a range of $0-54$ points, five items for depersonalization for $0-30$ points, and eight items for personal accomplishment for $0-48$ points. Conventional scoring indicates that each subscale is summed, reported separately, and designated as 'low' or 'high'. An EE score of $<17$ is considered low and $\geq 27$ is considered high. A depersonalization score $<7$ is considered low and $\geq 13$ is high. Inversely, a personal accomplishment score of $\geq 39$ is considered high and $\leq 31$ is low. According to Maslach and Jackson, scores that are both $>27$ on the EE subscale and $>13$ on the depersonalization subscale represent burnout. ${ }^{7}$

The 12-item Grit Scale is a reliable, validated instrument that assesses passion and perseverance using a fiveitem Likert scale for a range of scores of $12-60 .{ }^{17}$ The sum of all measured items is then divided by 12 and reported for an average participant score ranging from 1 through 5 that interprets 1 as 'not at all gritty' and 5 as 'very gritty'. ${ }^{17}$

\section{Procedure}

Pen and paper versions of the MBI-HSS and 12-Item Grit Scale were administered to the cohort in 2016 at the completion of Semester 6(Year 3), which signaled the end of the didactic portion of the problem-based curriculum. Participant reported that gender (as male or female) data were also collected alongside age in a range of 10-year increments. Repeated measures were collected just prior to program graduation in 2017 after the completion of clinical education experiences 3-4. Deidentified data were scored by hand by research assistants, verified by investigators, and stored in an excel spreadsheet. Only participant profiles that were complete with survey participation at both timeframes were analyzed. Any missing survey item data at baseline, Year 3, were replaced with an average item score for that item/measure for that participant at that time frame. Any missing survey item data at the repeated measure were managed with a last observation carried forward strategy where the first measure/trial data for that participant scored item were used. Statistical analysis was conducted using SPSS v. 25, and results were reported and interpreted on the MBI individual subscales consistent with supporting literature., ${ }^{7,10,11}$

Data analysis included descriptive statistics and factorial, and split-plot analysis of variance (ANOVA) with time and gender factors. Pairwise comparisons of simple effects were calculated using syntax. The alpha level was set at 0.05 for all interaction and main effects interpretations. The simple effects alpha level was adjusted to divide by two and interpreted at 0.025 to account for the two pairwise comparisons. Pearson's correlation coefficients were calculated between EE and depersonalization domain variables (that define burnout) and grit variables at both time periods with BCA bootstrapping applied in SPSS to get confidence interval ranges.

\section{Results}

Surveys were completed by 50 DPT students for a response rate of $100 \%$. Participants were $46 \%$ male and $54 \%$ female with all between the ages of 23 and 27 years old. Data met assumptions for the use of parametric statistical analysis. Descriptive data for burnout and grit, broken up by gender and cohort at Year 3 and graduation, can be found in Table 1. 
Table 1. Descriptive data: sample mean ( $\mathrm{Z}$ ), standard deviation ( $s$ ), and minimum (Min) and maximum (Max) values at curricular Year 3 (Y3) and graduation (Grad) for Maslach Burnout Inventory-Human Services Survey (MBI-HSS) subscales: emotional exhaustion (EE), depersonalization (DP), personal accomplishment (PA); Duckworth 12-Item Grit Scale (Grit).

\begin{tabular}{|c|c|c|c|c|c|c|c|c|c|}
\hline \multirow[t]{3}{*}{ Parameter } & \multirow[t]{3}{*}{ Gender } & \multicolumn{4}{|c|}{ Year 3} & \multicolumn{4}{|c|}{ Graduation } \\
\hline & & $\bar{\chi}$ & $s$ & Min & $\operatorname{Max}$ & $\bar{\chi}$ & $s$ & Min & Max \\
\hline & & Y3 & Y3 & Y3 & Y3 & Grad & Grad & Grad & Grad \\
\hline \multirow{3}{*}{$\begin{array}{l}\text { MBI-HSS } \\
\text { Subscale-EE }\end{array}$} & Male & 21.39 & 11.86 & 2.00 & 42.00 & 12.57 & 7.84 & 0.00 & 26.00 \\
\hline & Female & 15.14 & 9.96 & 2.00 & 38.00 & $|4.5|$ & 9.35 & 1.00 & 36.00 \\
\hline & Cohort & 18.02 & 11.21 & 2.00 & 42.00 & 13.62 & 8.65 & 0.00 & 36.00 \\
\hline \multirow{3}{*}{$\begin{array}{l}\text { MBI-HSS } \\
\text { Subscale-DP }\end{array}$} & Male & 5.48 & 3.68 & 0.00 & 14.00 & 4.00 & 3.74 & 0.00 & 13.00 \\
\hline & Female & 3.81 & 4.88 & 0.00 & 24.00 & 3.56 & 3.52 & 0.00 & 14.00 \\
\hline & Cohort & 4.58 & 4.40 & 0.00 & 24.00 & 3.76 & 3.59 & 0.00 & 14.00 \\
\hline \multirow{3}{*}{$\begin{array}{l}\text { MBI-HSS } \\
\text { Subscale-PA }\end{array}$} & Male & 38.95 & 5.82 & 26.00 & 48.00 & 43.70 & 3.97 & 33.00 & 48.00 \\
\hline & Female & 40.85 & 6.18 & 27.00 & 48.00 & 42.41 & 4.73 & 30.00 & 48.00 \\
\hline & Cohort & 39.98 & 6.02 & 26.00 & 48.00 & 43.00 & 4.40 & 30.00 & 48.00 \\
\hline \multirow[t]{3}{*}{ I2-Item Grit Scale } & Male & 3.91 & .32 & 3.25 & 4.58 & 4.03 & 0.35 & 2.83 & 4.50 \\
\hline & Female & 3.92 & .37 & 3.17 & 6.67 & 4.00 & 0.41 & 2.83 & 4.92 \\
\hline & Cohort & 3.91 & .35 & 3.17 & 4.67 & 4.02 & 0.38 & 2.83 & 4.92 \\
\hline
\end{tabular}

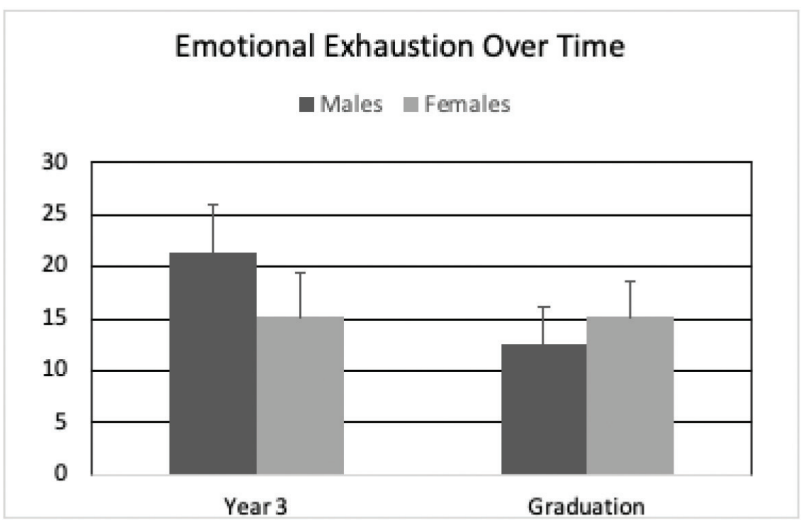

Fig. 1. Emotional exhaustion over time; interaction effect, $p=0.004$; confidence intervals represented with error bars.

\section{Emotional exhaustion}

Split-plot factorial two-way analysis of variance (ANOVA) revealed a significant interaction effect between gender $\times$ time on EE scores, $\mathrm{F}(1,48)=9.276, p=0.004$, see Fig. 1. Additional pairwise comparisons of simple effects indicated a significant effect of male gender on EE scores over time, $\underline{\chi}_{\text {diff }}=8.83, p=\leq 0.0001, d=0.896$; male scores significantly decreased from Year 3 to graduation. There were no significant differences between male and female student EE scores at each time frame: at Year 3, $\underline{\chi}_{\text {diff }}=6.24, p=0.049, d=0.572$, or at graduation, $\underline{\chi}_{\text {diff }}=1.95, p=0.432, d=0.227$. The main effect of gender on $\mathrm{EE}$ scores $\mathrm{F}(1,48)=0.768, p=0.385$ was not significant. The main effect of time on cohort EE scores was significant, $\mathrm{F}(1,48)=12.35, p=0.001, d=0.44$, showing a significant decrease in exhaustion levels for the cohort overall from Year 3 to graduation.

\section{Depersonalization}

There was a no significant interaction effect of gender $\times$ time on DP scores, $\mathrm{F}(1,48)=0.817, p=0.371$. The main effects of time, $\mathrm{F}(1,48)=1.659, p=0.204$, and gender, $\mathrm{F}(1,48)=1.32, p=0.256$, were also not significant.

\section{Personal accomplishment}

Interaction effects of gender $\times$ time on PA scores, $\mathrm{F}(1,48)$ $=3.408, p=0.07$, were not significant. The main effect of time on cohort PA scores, however, was significant, $\mathrm{F}(1,48)=13.322, p=0.001, d=0.58$; the cohort reported significantly higher personal accomplishment scores at graduation compared to Year 3 measures. The main effect of gender on PA scores was not significant, $\mathrm{F}(1,48)=$ $0.062, p=0.805$.

\section{Grit}

There were no significant interaction effects for gender on levels of grit regardless of time, $F(1,48)=0.144$, $p=0.706$. The main effect of time was significant, $\mathrm{F}(1,48)$ $=4.69, p=0.035, d=-0.28$; the cohort reported significantly higher grit scores at graduation compared to Year 3 ; The main effect of gender on Grit scores was not significant, $\mathrm{F}(1,48)=0.007, p=0.935$.

\section{Relationships between burnout and grit}

Correlations data for relationships between burnout and grit can be found in Table 2. A significant moderate inverse relationship existed between grit scores at Year 3 
Table 2. Relationships between burnout and grit.

\begin{tabular}{|c|c|c|c|c|c|c|}
\hline Variable & GritY3 & Grit Grad & EEY3 & DPY3 & EE Grad & DP Grad \\
\hline GritY3 & 1.00 & $\begin{array}{l}0.570 \text { *** }[0.342, \\
0.714]\end{array}$ & ns & ns & $\begin{array}{l}-0.447^{* *} \\
{[-0.686,-2 \mid 5]}\end{array}$ & $\begin{array}{l}-0.327^{*} \\
{[-0.539,-0.23]}\end{array}$ \\
\hline Grit Grad & & 1.00 & ns & ns & $\begin{array}{l}-0.528^{* * * *} \\
{[-0.717,-258]}\end{array}$ & $\begin{array}{l}-0.44 I^{* *} \\
{[-0.654,-102]}\end{array}$ \\
\hline EEY3 & & & 1.00 & $\begin{array}{l}0.540 * * * \\
{[0.280,0.730]}\end{array}$ & $\begin{array}{l}0.492 * * * \\
{[0.276,0.689]}\end{array}$ & $\begin{array}{l}0.482 * * * \\
{[0.292,0.647]}\end{array}$ \\
\hline DPY3 & & & & 1.00 & ns & $\begin{array}{l}0.309 * \\
{[-0.023,0.567]}\end{array}$ \\
\hline EE Grad & & & & & 1.00 & $\begin{array}{l}0.685^{* * *} \\
{[0.505,0.835]}\end{array}$ \\
\hline DP Grad & & & & & & 1.00 \\
\hline
\end{tabular}

Curricular Year 3 (Y3) and graduation (Grad) for Maslach Burnout Inventory-Human Services Survey (MBI-HSS) subscales: emotional exhaustion (EE), depersonalization (DP); Duckworth I2-Item Grit Scale (Grit).

ns $=$ not significant $(p>0.05),{ }^{*} p<0.05,{ }^{* *} p<0.01,{ }^{* * *} p<0.00 \mathrm{I} ; \mathrm{BCa}$, bootstrap $95 \% \mathrm{Cl}$ reported in brackets.

and EE scores at graduation, $r=-0.447, p=0.01, \mathrm{BCa}$ bootstrap CI $[-0.686,-0.215]$.

\section{Discussion}

Findings from this study indicate that EE scores for male students in the cohort were moderate at the end of the Year 3 timeframe and significantly declined over time, $p=0.0001, d=0.89$. However, there were no significant differences between male and female scores on any of the burnout subscales or grit scores at either of the timeframes studied, though gender differences in EE at Year 3 were very close to significance, $\underline{\chi}_{\text {diff }}=6.24, p=0.049$, $d=0.572$. As a cohort, this group of DPT students did not meet MBI criteria definition for burnout at either time; mean EE and personal accomplishment subscale scores did significantly change over time, however. There were, as expected, significant inverse relationships between grit at Year 3 and burnout at graduation.

While not statistically significant, EE at the end of didactic coursework was higher in males than females, which is counter to the findings of Williams et al., indicating higher levels of stress and exhaustion in female year 1 students. ${ }^{5}$ Our study looked at students further along in the PTEP (third-year students), and outcomes seem consistent with Williams' findings that second-year students reported being more stressed than their first-year counterparts. $^{5}$

The results of our study showed a significant, moderate inverse relationship between grit and EE over time. Our cohort's mean grit scores were higher (3.91) than that reported in recent pilot normative data of DPT students (3.78) and may be due to self-report instruments sensitivity to self-presentation and promotion. ${ }^{16,18,19,24}$ Even though the higher grit scores may have contributed to a stronger relationship in this small sample, our findings are consistent with other research suggesting a potential protective effect of grit on burnout. ${ }^{21}$

Students in this sample participated in 30 weeks of terminal clinical education. The Commission on Accreditation in Physical Therapy Education (CAPTE) reported from 2019 to 2020 data an average of 22 weeks reported in final terminal clinical education experiences. ${ }^{26}$ The impact of longer terminal experiences, where students are often away from their support network of the PTEP, family, and friends, on mental health and burnout is unknown. No known published studies have looked at the impact of physical therapy clinical education on professional (not academic) burnout in student physical therapists. It is unclear why male DPT students in this sample reported higher EE. Spataro et al. described female physicians as more likely to report EE and use maladaptive coping mechanisms like self-blame more often than their male counterparts, while Lopez-Lopez found that male gender positively correlated with burnout in mental health nurses..$^{27,28}$ We speculate that the small sample size, the use of self-reported instruments, and underlying social/demographic factors not collected may have influenced outcomes. Future study with larger samples across a variety of PTEP lengths is indicated.

This study was conducted with a small single class sample of age-homogenous students enrolled in a PTEP that uses a problem-based curricular model that may limit generalizability. This study did not control for differences in clinical education experiences, including schedule, workload, setting, and clinical instructor support, for example, which are potential confounding variables that may impact a student physical therapist's perceptions. ${ }^{9}$ A limited number of participant demographic variables were collected and analyzed. Additional demographic 
and programmatic data are needed to fully conceptualize the individual and work environmental factors predicting physical therapy student burnout.

Previous studies indicated relationships among gender, student loan debt, age, and other personal individual factors and rates of burnout in medical students. ${ }^{1}$ Correlation results in this study were significant, but effect sizes were low to moderate. Use of the MBI-HHS survey version instrument may not have appropriately described the burnout etiology participants experienced. Because third year DPT students in this study were responsible for both clinical practice schedules as well as online coursework, the different dimensions of academic burnout versus patient interaction burnout may have blurred results. Future study might consider using another version of the MBI (suited for medical professionals or students), a free OLBI version, or adapting a survey to appropriately capture the unique lived experience of the modern DPT student.

The timing of surveying students at baseline with questions geared toward constructs of work/professional burnout and grit before the start of clinical education may have confounded results. Additional data points may have been warranted to include participant baseline scores at the start of the program to appropriately represent a longitudinal design.

\section{Conclusion}

Male DPT students reported moderate levels of EE at the start of terminal clinical experiences that dropped significantly to low levels compared to female students. Student grit levels in this study were high compared to recent investigations of other DPT cohorts. There were significant inverse relationships between grit at the end of didactic training and burnout symptoms after clinical experiences, indicating a possible protective effect in DPT students.

Given the low correlation values, specific results for men, and single cohort design of this investigation, it appears that a broader study is needed to determine the nature of any relationship between grit and burnout and why men and women may be different. As a variety of PTEP delivery models and program lengths emerge, researchers can use burnout and grit screening tools geared toward specific time intervals and DPT student subpopulations to best understand the student experience during clinical education.

\section{Acknowledgments}

Romulo Montenegro, Michael Rosentreter, and Conner Williamson, Research Assistants and Student Physical Therapists at the time of this study, University of the Incarnate Word School of Physical Therapy, San Antonio, TX.

\section{Conflict of interest and funding}

No Funding. The authors have no conflicts of interest.

\section{Disclosures}

Portions of this content were presented at the American Physical Therapy Association's Educational Leadership Conference, October 2019, Bellevue, WA, as a poster, 'Levels of Grit in Entry-Level Doctor of Physical Therapy Students' and a platform presentation, 'Levels of Burnout in Entry-Level Doctor of Physical Therapy Students Pre-Post Extended Clinical Education'.

\section{Ethical approval}

Burnout and grit survey instruments were added to the School of Physical Therapy programmatic teaching and learning IRB, Learning Outcomes of a Problem Based Learning Doctor of Physical Therapy Program (\#12-08003 ) as an addendum and approved for use. Institutional Review Board, University of the Incarnate Word, San Antonio, TX.

\section{References}

1. West CP, Dyrbye LN, Shanafelt TD. Physician burnout: contributors, consequences, and solutions. J Intern Med 2018; 283: 516-29. doi: 10.1111/joim.12752

2. Dewa CS, Loong D, Bonato S, Trojanowski L. The relationship between physician burnout and quality of healthcare in terms of safety and acceptability: a systematic review. BMJ Open 2017; 7 : e015141. doi: 10.1136/bmjopen-2016-015141

3. Williams ES, Manwell LB, Konrad TR, Linzer M. The relationship of organizational culture, stress, satisfaction, and burnout with physician-reported error and suboptimal patient care: results from the MEMO study. Health Care Manage Rev 2007; 32: 203-12. doi: 10.1097/01.HMR.0000281626.28363.59

4. Bullock G, Kraft L, Amsden K, et al. The prevalence and effect of burnout on graduate healthcare students. Can Med Educ J 2017; 8: e90-108. https://doi.org/10.36834/cmej.36890

5. Williams PS, Mueller MK, Carroll HC, Cornwall MW, Denney LM, Kroneberger LM. Patterns of academic burnout, emotional distress, and coping in physical therapy students. Int $\mathrm{J}$ Health, Wellness Soc. 2018; 8: 31-46. doi: 10.18848/2156-8960/ CGP/v08i03/31-46

6. Macauley K, Plummer L. Prevalence and predictors of anxiety in doctor of physical therapy students. J Allied Health 2017; 46: e39-41.

7. Maslach C, Jackson SE, Leiter MP. Using the MBI-Human Services Survey. In: Maslach C, Jackson SE, eds. Maslach Burnout Inventory Manual. Menlo Park, CA: Mind Garden, Inc; 1996, pp. 4-17.

8. Halbesleben JRB, Demerouti E. The construct validity of an alternative measure of burnout: investigating the English translation of the Oldenburg Burnout Inventory. Work \& Stress 2005; 19: 208-20. doi: 10.1080/02678370500340728

9. Struessel T, Forster JE, Van Zytveld C. Doctor of physical therapy student experiences during clinical education: patient safety, error, and the system. J Phys Ther Educ 2019; 33: 3-15. doi: 10.1097/JTE.0000000000000072

10. Berry JW. A regional survey and analysis of burnout among physical therapists in frontier counties. Phys Ther J Policy Admin Leadership 2015; 15: J3-J11.

11. Balogun JA, Titoloye V, Balogun A, Oyeyemi A, Katz J. Prevalence and determinants of burnout among physical and occupational therapists. J Allied Health 2002; 31: 131-139. 
12. Robertson HD, Elliott AM, Burton C, et al. Resilience of primary healthcare professionals: a systematic review. Br J Gen Pract 2016; 66: e423-33. doi: 10.3399/bjgp16X685261

13. Mueller K, Prins R, de Heer HD. An online intervention increases empathy, resilience, and work engagement among physical therapy students. J Allied Health 2018; 47: 196-203.

14. Halbur B, Kopp A. Grit of first and second-year physical therapy students: a pilot study. Phys Ther Schol Project 2019; 664. Available from: https://commons.und.edu/pt-grad/664/ [cited 30 August 2019].

15. Roll M, Canham L, Salamh P, Covington K, Simon C, Cook C. A novel tool for evaluating non-cognitive traits of doctor of physical therapy learners in the United States. J Educ Eval Health Prof 2018; 15: 1-8. doi: 10.3352/jeehp.2018.15.19

16. Bliss R, Jacobson E. Doctor of physical therapy student grit as a predictor of academic success: A pilot study. Health Prof Educ 2020; 6: 1-7. doi: 10.1016/j.hpe.2020.06.006

17. Duckworth AL, Peterson C, Matthews MD, Kelly DR. Grit: Perseverance and passion for long-term goals. J Pers Soc Psychol 2007; 92: 1087-101. doi: 10.1037/0022-3514.92.6.1087

18. Richardson S, Scotto M, Belcina M, Patel R, Wiener K. Grit, and academic performance in doctor of physical therapy students. Internet J Allied Health Sci Pract 2020; 18: 1-4.

19. Huhn K, Brittney Rusinski DPT, Amanda Saucier DPT, et al. Exploration of grit and emotional intelligence and success in a doctor of physical therapy program. Internet J Allied Health Sci Pract 2021; 19: 1-7.

20. Carp S, Fry K, Gumerman B, Pressley K, Whitman A. Relationship between grit scale score and academic performance in a doctor of physical therapy program: a case study. J Allied Health 2020; 49: 29-35.

21. Jumat MR, Chow PK-H, Allen JC Jr, et al. Grit protects medical students from burnout: a longitudinal study. BMC Med Educ 2020; 20: 1-9. doi: 10.1186/s12909-020-02187-1

22. Cortez AR, Winer LK, Kassam A-F, et al. Exploring the relationship between burnout and grit during general surgery residency: a longitudinal, single-institution analysis. Am J Surg 2020; 219: 322-7. doi: 10.1016/j.amjsurg.2019.09.041

23. Van Veld R, Slaven EJ, Reynolds B, Shupe P, Woolery C. Firstyear doctor of physical therapy students demonstrate change in coping with stress. J Phys Ther Educ 2018; 32: 138-44. doi: 10.1097/JTE.0000000000000037

24. Niessen ASM, Meijer RR, Tendeiro JN. Measuring non-cognitive predictors in high-stakes contexts: the effect of self-presentation on self-report instruments used in admission to higher education. Pers Individ Dif 2017; 106: 183-9. doi: 10.1016/j. paid.2016.11.014

25. Duckworth AL, Yeager DS. Measurement matters: assessing personal qualities other than cognitive ability for educational purposes. Educ Res 2015; 44: 237-51. doi: 10.3102/0013189X15584327

26. The Commission on Accreditation in Physical Therapy Education. Aggregate program data 2019-2020: Physical therapist education programs fact sheet. Published March 2020. Available from: http://www.capteonline.org/uploadedFiles/CAPTEorg/ About_CAPTE/Resources/Aggregate_Program_Data/AggregateProgramData_PTPrograms.pdf [cited 01 February 2021].

27. Spataro BM, Tilstra SA, Rubio DM, McNeil MA. The toxicity of self-blame: sex differences in burnout and coping in internal medicine trainees. J Women's Health 2016; 25: 1147-52. doi: 10.1089/jwh.2015.5604

28. López-López IM, Gómez-Urquiza JL, Cañadas GR, et al. Prevalence of burnout in mental health nurses and related factors: a systematic review and meta-analysis. Int J Ment Health Nurs 2019; 28: 1032-41. doi: 10.1111/inm.12606

* Jennifer C. Kish

104 Health and Human Services Blvd

Bowling Green, $\mathrm{OH}$ 43403, USA

Email:kishjc@bgsu.edu 